\title{
Lumen
}

Selected Proceedings from the Canadian Society for Eighteenth-Century Studies

\section{La Preuve par Jean-Jacques : un « fou littéraire » juge Rousseau}

\section{Tanguy L'Aminot}

Volume 15, 1996

URI : https://id.erudit.org/iderudit/1012474ar

DOI : https://doi.org/10.7202/1012474ar

Aller au sommaire du numéro

Éditeur(s)

Canadian Society for Eighteenth-Century Studies / Société canadienne d'étude du dix-huitième siècle

ISSN

1209-3696 (imprimé)

1927-8284 (numérique)

Découvrir la revue

Citer cet article

L'Aminot, T. (1996). La Preuve par Jean-Jacques : un " fou littéraire » juge

Rousseau. Lumen, 15, 71-83. https://doi.org/10.7202/1012474ar d'utilisation que vous pouvez consulter en ligne.

https://apropos.erudit.org/fr/usagers/politique-dutilisation/ 


\section{La Preuve par Jean-Jacques: Un 'fou littéraire' juge Rousseau}

La notion de fou littéraire est doublement imprécise. Elle l'est par son premier terme, puisque le mot de folie recouvre des processus très différents, par leur cause, leur expression, leur gravité et leur évolution. Le cas que nous allons étudier se rapproche assez de celui de Rousseau, tel que le présente le docteur Jacques Borel dans Génie et folie de J.-J. Rousseau: il parle en effet d'une schizophrénie qui se traduit par 'un ensemble de convictions morbides plus ou moins organisées en système, sur un thème unique de persécution; l'intelligence reste normale. Le délire évolue par extension progressive dans l'espace et le temps: les points d'action de la malveillance se multiplient, dans une ubiquité qui défie toute vraisemblance, et plus ou moins vite le sujet y intègre les événements passés, dans une marche anachronique. La conviction est absolue et le raisonnement n'a aucune prise sur elle.' Le docteur Borel décrit la faculté d'interprétation du schizophrène qui le conduit à donner un sens à tout ce qui se passe et à tout rapporter à lui ‘sous le coup d'une causalité préméditée et universelle, dans une atmosphère de mystère, de manoeuvres, de manigances le plus souvent inexplicables. ${ }^{1}$

Le second terme, le qualificatif 'littéraire,' ne simplifie pas la question. Raymond Queneau a tenté de donner quelques éclaircissements sur l'expression 'fou littéraire' dans son roman Les Enfants du limon, paru en 1938. Le personnage de ce livre, Chambernac, envisage d'écrire 'un grand ouvrage sur la question qui serait à la fois une biographie, une bibliographie et une anthologie de tous les fous littéraires français du XIXe siècle.' Se fondant sur Charles Nodier, Octave Delepierre et quelques auteurs qui se sont intéressés aux écrivains singuliers et oubliés, il pose après divers tâtonnements qu'un fou littéraire est 'un auteur imprimé dont les élucubrations (je n'emploie pas ce mot péjorativement) s'éloignent de toutes celles professées par la société dans laquelle il vit, soit par cette société dans son ensemble, soit par les différents groupes, même minimes, qui la composent, ne se rattachent pas à des doctrines antérieures et de plus n'ont eu aucun écho. Bref, un 'fou littéraire' n'a ni maîtres ni disciples' et doit rester inconnu. Queneau ajoute aussi que, pour bénéficier de cette appellation, le fou littéraire doit 
écrire 'des choses qu'un individu moyen, comme moi, considère comme excessivement singulières. ${ }^{2} \mathrm{Il}$ admet que cette définition ne convient pas toujours et cite divers cas qui la contredisent. André Blavier, qui a réalisé depuis le projet de Chambernac et publié en 1982 une volumineuse anthologie des fous littéraires, se range à cet avis et rappelle un autre texte de Queneau où celui-ci expose combien il est délicat de porter sur un homme 'un jugement d'aliénation mentale uniquement d'après la forme et le contenu de ses écrits. ${ }^{3}$ Une telle approche conduit à ranger maints novateurs dans cette catégorie: P.G. Brunet n'y avait pas manqué qui, en 1880, avait classé sous cette étiquette William Blake, Sade, Rétif, Michelet, Nerval, Mickiewicz, Walt Whitman et bien d'autres écrivains célèbres sous prétexte que leurs écrits présentent quelques bizarreries. ${ }^{4}$

Notre propos ne sera pas de résoudre ces difficiles questions, mais simplement de présenter un auteur qui a lu et utilisé Rousseau de manière très particulière, un auteur que Raymond Queneau et André Blavier ont classé sans ambiguité dans la catégorie des fous littéraires: Pierre Marie Théodore Choumara. ${ }^{5}$ Le Larousse du XXe siècle donne à son propos la notice suivante: 'Officier français du Génie né à Nonancourt en 1787, mort en 1870. Ses réformes dans l'art de la fortification portent le nom de système de Choumara. Il a proclamé l'indépendance des escarpes et des parapets, et inventé un fourneau de cuivre encore en usage dans les troupes.' Pour nous, il est surtout l'auteur d'une suite des Confessions publiée en 1841: Le Fou du roi, ou Confessions d'un ingénieur militaire formant commentaires et complément aux Confessions de J.-J. Rousseau./Preuves évidentes de la conjuration dont ce grand homme parle dans ses écrits; Véritables auteurs de cette conjuration;/ Abominables moyens employés pour le torturer pendant sa vie, pour l'empêcher d'écrire et qui ont causé sa mort;/ Reproduction de ces moyens, depuis la Révolution, contre d'autres individus [...];/ Influence de la police jésuitique sur l'affaiblissement progressif de la France, sur les crimes et fléaux qui l'ont épouvantée;/ Moyens de prévenir le retour de ces calamités;/Solution du plus grand problème social de l'époque, etc.

Avant de présenter et d'analyser le commentaire et l'utilisation que Choumara fait de Rousseau, arrêtons-nous un instant pour donner quelques précisions biographiques indispensables. Celles-ci sont fournies par Choumara lui-même qui, dans Le Fou du roi, Théodore et plusieurs autres écrits, rappelle les principales étapes de son existence et de sa persécution, et les interprète en fonction de sa situation présente, selon le processus classique de la folie raisonnante.

Il est né en 1787 dans des conditions douloureuses, puisqu'il a eu un pied tourné et a dû porter dans son enfance un appareil pour le redresser. En 1794, il est choisi pour lire la Déclaration des droits de l'homme à la fête de l'Etre suprême. Il fait ensuite de brillantes études qui le conduisent à l'Ecole polytechnique à dix-sept ans. A sa sortie, il devient officier 
dans le corps du Génie et participe aux diverses campagnes napoléoniennes. De 1810 à 1814, il fait partie de l'armée qui mène la guerre au Portugal et en Espagne, et défend notamment la place d'Astorga. Il est fait prisonnier, s'évade et rejoint Paris après la chute de l'Empereur. On le retrouve à l'état-major du Génie du duc de Berry dans les premiers jours de mars 1815, mais quand Napoléon arrive à Paris, le 20, il le rejoint et participe en juin à la bataille de Waterloo où il est grièvement blessé.

En février 1816, il fait cependant partie des armées de la Restauration. Il ne renie pas pour autant l'Empereur et il a alors, à ce propos, une violente altercation avec un autre officer, Saint-Marcellin. C'est à cet épisode qu'il remonte pour trouver l'origine de ses malheurs et l'arrêt de son avancement dans la carrière militaire, Saint-Marcellin étant protégé par le maréchal Soult, avec lequel Choumara sera plus tard en mauvais termes. Forcé de quitter l'armée et d'être demi-solde pendant quelques mois, il est rétabli dans son grade et envoyé à Bayonne en automne 1816. Commence alors un périple à travers la France des garnisons, avant de rejoindre Paris en 1826. C'est à cette date qu'il publie ses premiers livres: Considérations sur les effets de l'artillerie dans la défense des places et Premier mémoire sur la fortification. C'est aussi à cette date qu'il entre en polémique avec le général Haxo qu'il accuse de lui avoir volé sa théorie sur l'art des fortifications: il s'empresse d'éditer mémoires et observations afin de s'en assurer la propriété.

Quand survient la révolution de 1830, il s'enthousiasme du renversement du régime de Charles $X$, mais sa participation se réduit à la rédaction d'une proclamation destinée à Louis-Philippe et réclamant plus de justice pour les anciens élèves de l'école polytechnique. En novembre 1831, Choumara démissionne de l'armée: il est depuis dixneuf ans capitaine et ses mérites ne sont pas reconnus. Il constate que d'autres officiers ayant moins d'ancienneté et de valeur passent plus rapidement au grade supérieur et en éprouve de l'amertume. Ces éléments-là lui serviront plus tard à étayer la thèse du complot, dont il se sent victime. En 1831, il publie également ses Lettres au ministre de la guerre sur les fourneaux des casernes, la fortification et les mines, qui lui vaudront une certaine notoriété, mais l'année suivante, en 1832, il plaide contre le ministre de la guerre, le maréchal Soult, qu'il accuse de lui avoir volé son invention concernant un nouveau système de fourneaux. Il poursuit néanmoins ses recherches et rédige plusieurs nouvelles brochures sur les fortifications, engage un travail historique sur les campagnes napoléoniennes et la bataille de Toulouse qui opposa en 1814 Soult à Wellington. Cela aboutit à plusieurs articles qui paraissent de 1835 à 1838 dans le Journal des sciences militaires et sont rassemblés ensuite pour former un fort volume qui paraît en 1840. Ces dates ont leur importance, 
car elles permettront à Choumara de fonder par la suite sa théorie du complot britannique contre la France et contre lui.

En 1839, il a d'ailleurs réintégré l'armée (il affirme dans Théodore que le ministre de la guerre lui a écrit personnellement en 1834 pour le lui proposer) et il est enfin nommé chef de bataillon en février 1840 .

C'est à cette époque aussi que sa vie bascule dans le drame. Au début de 1839 , il vit rue des Petits-Augustins à Paris, mais six mois après, trois locataires emménagent en même temps sur le même palier que lui: il les soupçonne aussitôt d'être des agents chargés de le surveiller. Il démenage quai Saint-Michel, dans un logement occupé autrefois par un journaliste libéral et équipé, selon lui, d'un système secret permettant de surveiller le locataire. La maison qu'il possède à Nonancourt, dans l'Eure, est cambriolée. Tous ces événements amènent Choumara à se plaindre au préfet de Police de Paris dans deux lettres où il cherche à comprendre ce qui lui arrive et qu'il édite peu après. Il affirme qu'on le surveille depuis fort longtemps et remonte jusqu'en 1816 et à son duel avec Saint-Marcellin. Malgré les lettres et les plaintes adressées au commissaire du quartier, il est persuadé que quelqu'un pénètre chez lui régulièrement et prend conscience que son propriétaire et les fonctionnaires de police le regardent comme un insensé. Dans la seconde lettre au Préfet (16 août 1840), Choumara expose que ces malveillances sont dues à des policiers subalternes et demande à leur chef de les faire cesser. Il affirme aussi que ces faits lui 'donnent un point de ressemblance avec l'illustre et malheureux J.-J. Rousseau, qui a été le jouet et la victime de toutes les polices de $\mathrm{l}^{\prime}$ Europe.${ }^{6}$ Choumara déménagera trois fois encore encore de 1840 à 1842 sur la rive droite de la Seine, mais le 13 mars 1843, il est de nouveau l'objet d'un attentat pendant son sommeil. Cette agression lui permet de découvrir, en poussant les recherches jusque dans l'immeuble voisin où il loue une chambre, un passage secret qui conduit chez lui. Il a désormais une preuve concrète de ce qu'il avance depuis des années. En 1845 cependant, il en est encore à écrire des lettres au Président du Conseil (lettres qui constituent la première édition de Théodore - 1846) pour rappeler tous les épisodes survenus et les interpréter dans le cadre du complot qu'il imagine. L'année suivante, il adresse même une pétition à la Chambre des députés pour dénoncer une fois de plus l'action de la police secrète.

Car Choumara, s'il pense être la cible d'un complot qui le dépasse et sur lequel il n'a pas de prise, essaie de trouver un sens à ce qui lui arrive dans plusieurs livres qu'il publie de 1841 à 1847, ces publications étant menées parallèlement avec les études consacrées aux fortifications et aux guerres de l'Empire.

Le premier de ces ouvrages est $L e$ Fou du roi qui paraît en 1841. Il s'agit d'un livre de 144 pages, composé de six chapitres, dans lesquels Chou- 
mara place son aventure dans une perspective politique et historique plus générale. Son argumentation repose essentiellement sur l'analogie de son cas avec celui des 'hommes de tête et de coeur' de son temps, mais aussi avec celui de Rousseau chez qui il voit un précurseur. Si, en 1840, il pensait être la victime de quelques policiers subalternes incontrôlés, il est persuadé un an après que son persécuteur n'est autre que la police secrète qui agit selon son bon plaisir et de façon totalement illégale. Le second chapitre du livre est consacré à révéler ses méthodes à travers l'histoire d'un capitaine d'artillerie qui ressemble beaucoup à Choumara, a connu une aventure semblable à la sienne et s'est trouvé enfermé dans un asile. Choumara peint les membres de cette police sous le jour le plus sombre: elle est composée selon lui des plus grands scélérats, de criminels et de prostituées, de 'ces viveurs de nuit, ignobles étalons soldés et dressés pour la chasse aux cuisinières et aux femmes de chambre, afin de dérober les secrets les plus intimes des familles. ${ }^{8}$ Cette caste d'individus louches, assez puissante pour agir impunément, assez riche pour payer des complices et assez redoutable pour imposer un secret inviolable, diffère du modèle traditionnel du barbouze tel que nous l'imaginons à travers les romans et les films policiers, en ce sens que, selon Choumara, elle étend son empire non seulement sur la France, mais sur le monde entier. ${ }^{9}$ Il s'agit en effet, dans son esprit, des jésuites qui, revenus en France après la Restauration, en 1814, manoeuvrent pour imposer leur pouvoir dans tous les cercles de la société et se débarrasser de leurs ennemis. Une telle 'révélation' nous montre comment la paranoïa de Choumara utilise à son profit l'actualité politique de son temps, et particulièrement la lutte engagée alors par les libéraux contre les jésuites: dès 1826 s'était répandu le bruit d'une conspiration jésuitique au point que l'affaire avait été portée à la Chambre. En 1828, la correspondance secrète des jésuites avait été révélée et les Pères avaient dû abandonner les huit collèges d'enseignement que le roi leur avait attribués en 1814. Cette lutte qui trouvera en 1843 et en 1845 son apogée, avec la publication du pamphlet de Jules Michelet et Edgar Quinet, Des Jésuites, puis avec une nouvelle ordonnance contre la Compagnie de Jésus, fournit à Choumara l'explication de ce qui lui arrive. Les ministères, l'armée et particulièrement la police sont infestés par les 'jésuites de robe courte' — c'est-à-dire par les affiliés que dénonce la presse libérale - qui ne reculent ni devant le crime ni devant la subversion politique. Choumara cite ainsi les noms de plusieurs officiers dont la mort suspecte apparaît comme leur oeuvre et accuse Guizot d'avoir été nommé à la tête du gouvernement en échange de sa soumission à leurs desseins. Le Fou du roi, Théodore et d'autres livres de Choumara traduisent aussi une haine violente à l'égard de l'Angleterre. L'ancien soldat de Bonaparte exprime une anglophobie assez courante à l'époque 
chez les Français, mais qui s'adapte parfaitement à son image du complot: les jésuites qui l'espionnent, travaillent pour le compte de la Grande-Bretagne, à qui ils livrent le résultat de ses recherches sur les fortifications et les fourneaux. ${ }^{10}$

C'est au sein de ce 'raisonnement' que prend place le discours de Choumara sur Rousseau. Pour cet officier bonapartiste, attaché aux principes de la Révolution et de l'Empire, le Citoyen de Genève apparaît comme un précurseur qui a vécu la même aventure que lui: ils ont connu les mêmes ennemis et sont victimes des mêmes méthodes. Choumara trouve dans Les Confessions et les Dialogues la preuve de ce qu'il avance. Grâce à Jean-Jacques, il prouve qu'il est sain d'esprit et par contrecoup son aventure prouve la véracité des propos de Rousseau quand il dénonçait le 'complot des ténèbres,' et annule d'un trait toute la littérature consacrée depuis le XVIIIe siècle à la folie du philosophe. Cette littérature - Claude Wacjman l'a fort bien montré - a été régulière et dispersée depuis le moment où les contemporains de Rousseau notaient ses bizarreries, mais c'est à partir des études rétrospectives de Mme de Staël qu'on est passé d'une représentation de Rousseau fou à une représentation de la folie de Rousseau qui touchait à sa pensée philosophique et à ses écrits. 'Que Rousseau ait été fou, au sens familier du terme: bizarre, comme il a été écrit, cela ne fait pas de doute,' commente Claude Wacjman:

Qu'il ait été considéré comme atteint de folie, comme malade mental, comme aliéné de son vivant et au sens où nous l'entendons actuellement, cela est beaucoup plus discutable, et peu d'indices et de témoignages directs vont dans ce sens. Ceux des plus virulents viennent de ses opposants, de ceux qui, tel Voltaire, seront décrits, comme étant ses ennemis. Il y avait état de guerre, qui appelait à se défendre, il n'y avait pas ostracisme par peur d'une contagion possible de cette folie qui, en tant que telle ne s'est manifestée qu'après qu'elle ait été reconnue de diverses manières. ${ }^{11}$

Vers 1840, quand Choumara publie Le Fou du roi et Théodore, cette évolution se confirme qui aboutira à la fin du XIXe et au début du XXe siècles aux thèses des médecins littéraires sur la maladie et la psychologie de Rousseau.

Le discours de Choumara est donc une remise en cause totale de cette évolution, même s'il demeure marginal et ignoré. Commentant la sixième lettre du livre de Mme de Staël (Lettres sur les ouvrages et le caractère de J.-J.Rousseau - 1788), Choumara écrit:

L'auteur de Corinne était sans doute digne de comprendre l'auteur de Julie, et pourtant elle le défigure, quelle profanation dans cette épithète d'insensé ap- 
pliquée aux Dialogues dans lesquels Rousseau s'est peint avec encore plus de perfection que dans ses Confessions, et dans lesquels il a rassemblé toutes les combinaisons et tous les renseignements qui pouvaient mettre la postérité sur la voie pour découvrir les trames ténébreuses ourdies contre l'homme de génie qui consacre sa vie à la défense de la vérité. Ah! madame de Staël, avec quel empressement vous rétracteriez ce blasphème si vous pouviez voir les preuves de la réalité des faits avancés par l'auteur que vous appréciez si bien sous d'autres rapports. ${ }^{12}$

Voir! c'est de nouveau le problème fondamental: comment percevoir la réalité de Rousseau alors que les commentateurs du philosophe se sont placés 'sur le chemin de l'interprétation, non pas de la réalité, mais plutôt des perceptions que l'on a élaborées depuis la réalité. ${ }^{13}$ La méthode de Choumara est fondée sur l'analogie qui existe, selon lui, entre Jean-Jacques et lui: c'est parce qu'il vit la même réalité que Rousseau qu'il peut dévoiler la vérité jusqu'ici cachée ou étouffée. Le Fou du roi commence par une ode à Rousseau qui souligne ce rapport:

Comme toi, solitaire au milieu de Paris, par suite de mon amour pour la méditation, la justice et la vérité;

Comme toi, indigné de la bassesse des monstres qui, en te torturant et t'empoisonnant, $t^{\prime}$ ont condamné à un repos prématuré et ont étouffé les chefs-d'oeuvre que recélaient encore ta brillante imagination et ton noble coeur; [...]

Comme toi, opposant un front calme et serein et le regard écrasant du mépris, à leur masque ricaneur et traître, je suis passé par toutes les impressions que tu as éprouvées, moins celle de la crainte; dès lors je t'ai compris, ta conduite m'a été expliquée; tes fautes, tes contradictions apparentes, se sont évanouies comme les ombres de la nuit devant les rayons du soleil. Je puis être ton interprète, puisque je vois ce que tu as vu, puisque, placé dans des circonstances à peu près semblables, $j^{\prime}$ agis à ta manière sans autre différence que celle résultant des temps, des institutions et des positions respectives. ${ }^{14}$

Dans l'édition de 1847 de Théodore, Choumara consacre pareillement un chapitre à montrer cette analogie des situations et des persécutions. S'appuyant sur plusieurs extraits des Confessions et des Dialogues. il affirme que 'la police anglo-gallo-jésuitique veut le traiter à la J.-J. Rousseau. ${ }^{15}$ Que les jésuites soient, d'après lui, leur ennemi commun assure le passage d'un siècle à l'autre et donne plus de cohésion à son propos.

Choumara n'en est pas moins conscient de la supériorité de Rousseau quant au génie, mais, dit-il, 
je ne crains pas d'accoler mon nom à ton grand nom, ni de rester au-dessous de la noble tâche que j'entreprends. Si je n'ai point, comme toi, la magie du style pour donner de l'intérêt aux choses les plus communes et les plus triviales en apparence, au moins, je ne suis pas dépourvu de la chaleur de coeur qui pousse les hommes généreux vers les nobles entreprises. ${ }^{16}$

Il trouve en fait autant d'importance à ses travaux sur les fortifications et les fourneaux qu'aux écrits de Rousseau: il y voit le bénéfice retiré par la patrie et l'origine de conflits internationaux. Cette identification à Rousseau qui finit par le rendre plus important que son modèle, est l'un des signes les plus évidents de la folie: on la retrouve d'ailleurs chez un autre 'fou littéraire,' Jean-Baptiste Roussy-Roussy qui, en 1874, prétendit être le sosie de Rousseau. Grâce à un prospectus de souscription qui en donne le plan, on sait qu'il eut le projet de publier un livre sur ce sujet: Le Sosie de J.-J. Rousseau, ou la reproduction matérielle et accidentelle de cet homme illustre, au moral, au physique et en psychologie. ${ }^{17}$

L'identification de Choumara diffère sensiblement de celles des auteurs étudiés par Jean Roussel. ${ }^{18}$ Comme eux, il connaît certes son Rousseau sur le bout des doigts et semble avoir étudié ses écrits, comme en témoignent les citations des Confessions, des Dialogues, des Rêveries et même du Contrat Social, dont les références sont données en note. Il cite également quelques contemporains comme Grimm, Voltaire ou Corancez et des commentateurs comme Mme de Staël et Musset-Pathay, mais s'identifier à Rousseau ne consiste pas pour lui à justifier sa solitude ou à s'évader vers un monde meilleur. Il n'y a pas tant analogie de sentiments - analogie qui a conduit les auteurs comme Louis-Claude de Saint-Martin ou Chateaubriand à déterminer dans leur mémoire, à partir des Confessions, 'des zones où règne le repos du souvenir, ${ }^{, 19}$ qu'analogie des situations. La lecture de Choumara reste centrée sur son idée fixe: prouver la santé mentale de Rousseau pour prouver la sienne.

Plusieurs citations du philosophe reviennent régulièrement sous sa plume qui traduisent son obsession et sa fixation. Dans Le Fou du roi et Théodore, il rappelle à plusieurs reprises ce passage du livre VII des Confessions:

Les planchers sous lesquels je suis ont des yeux, les murs qui m'entourent ont des oreilles, environné d'espions et de surveillants malveillants et vigilants, inquiet et distrait, je jette à la hâte sur le papier quelques mots interrompus qu'à peine j'ai le temps de relire, encore moins de corriger. Je sais que malgré les barrières immenses qu'on entasse sans cesse autour de moi l'on craint toujours que la vérité ne s'échappe par quelque fissure. ${ }^{20}$ 
Choumara se contente de souligner certains groupes de mots qui renvoient pour lui à une réalité quotidienne. En témoigne aussi un extrait des Dialogues qu'il cite volontiers, celui où Rousseau évoque la façon dont 'les murs, les planchers, les serrures' sont disposés autour de lui pour mieux l'espionner et comment 'les barboteuses de ces messieurs' prennent des airs de vierge pour $\mathrm{l}^{\prime}$ aborder. ${ }^{21}$ Son commentaire de Rousseau est plus technique que littéraire et consiste plus à expliquer le mécanisme de tel passage secret ou les vertus de tel poison ou somnifère qu'à apprécier le rythme de la Cinquième promenade.

Choumara a la certitude que la police utilise les mêmes méthodes d'un siècle à l'autre. Lui aussi est victime des 'barboteuses.' L'érotomanie qui est souvent associée à la paranoïa, se révèle chez lui quand il affirme que les jésuites sont des saint-simoniens et prônent la communauté des femmes:

Cette communauté des femmes existe par le fait dans la police secrète, où toutes les volontés sont brisés pour le salut de l'ordre, toutes les filles publiques, entretenues ou autres ne sont-elles pas la propriété des espions secrets? N'en changent-ils pas à volonté? Dans leurs orgies, payées par les fonds secrets, ne se forme-t-il pas un pêle-mêle où chacun prend ce qui lui tombe sous la main? Leurs maîtresses, leurs femmes même ne sont-elles pas livrées par eux aux hommes dont ils veulent pénétrer les secrets ou qu'ils veulent faire tomber dans leurs pièges, de même que leurs étalons sont détachés après les femmes dont la corruption leur est nécessaire? ${ }^{22}$

La folie de Choumara se révèle ici positive, car elle rompt avec l'image de Thérèse Levasseur laissée par Boswell, Grimm ou Mme de Staël. A ceux-ci qui lui ont reproché d'être une dévergondée, Choumara réplique qu'elle fut contrainte de trahir Rousseau, et qu'elle est plus à plaindre qu'à blâmer: une telle attitude ne se trouvera pas chez les commentateurs de Rousseau avant la seconde moitié du XXe siècle. Choumara explique:

Elle était forcée de se livrer aux espions, de se laisser faire des enfants par eux, afin qu'on pût les attribuer à Rousseau et que l'odieux de leur dépôt aux enfants trouvés reposât sur lui.

Elle était forcée de recevoir d'eux des maladies de toute espèce afin de les transmettre au bienfaiteur qui l'avait tirée de la misère et de l'avilissement où une première séduction l'avait plongée. ${ }^{23}$

Voilà résolue la difficile question des enfants de Rousseau, et du même coup celle de sa maladie et de sa mort. Prenant appui sur le fait que Rousseau dit dans les Dialogues qu'on le regarde comme 'un pestiféré,' Choumara explique avec force détails techniques dus à ses lectures et à 
son expérience personnelle, la manière dont Rousseau a été empoisonné. Les jésuites en sont les assassins puisqu'un poison porte le nom de 'fève de Saint-Ignace': ils ont jeté sur le philosophe, comme ils l'ont fait sur Choumara, une pousiière pestilentielle qui créait des démangeaisons et écartait de lui ceux qui le croisaient quand il sortait, les confirmant dans l'idée que Jean-Jacques était un pestiféré. C'est pour se protéger que, selon lui, Rousseau adopta le costume d'Arménien. Thérèse Levasseur a été manipulée par eux.

Si cette version ajoute en rocambolesque à celles qui avaient déjà transformé la mort de Rousseau en roman policier, selon l'expression de Ralph A. Leigh, le raisonnement de Choumara perd toute crédibilité quand il soutient que Rousseau était sous la surveillance des jésuites dès 1746. Il attira leur attention à l'époque de sa conversion au catholicisme, à Turin et ses ennemis ne lui ont pas pardonné le retour à la foi de ses ancêtres en 1755. On a cependant du mal à comprendre pourquoi Thérèse était dès la conception de son premier enfant, en 1746, soumise au pouvoir libidineux des jésuites, puisqu'à cette époque Rousseau travaillait pour Madame Dupin, s'embourgeoisait gentiment et ne menaçait par aucun écrit ni l'ordre ni la religion. Sans doute, les jésuites étaient-ils prévoyants.

L'aventure de Rousseau en Grande-Bretagne est aussi l'occasion d'une importante révélation. Choumara est certain du complot et cite la lettre de David Hume à M. de Guerchy, des passages de Voltaire, Grimm et Corancez pour expliquer comment on a essayé de faire passer Rousseau pour fou auprès des autorités et du public. Ainsi, 'on le mettait à la discrétion de la police administrative qui pouvait à son gré, sans jugement, sans bruit, le placer par grâce, dans quelque asile éloigné de la capitale où l'on n'entendrait plus parler de lui, ou bien le renfermer dans une maison d'aliéné, où il serait facile de le rendre tel. ${ }^{24}$ Rousseau a su éviter les pièges et ses ennemis ont alors tenté de l'empoisonner. Pour preuve suprême, Choumara évoque la lettre adressée par Rousseau au général Conway, en mai 1767, dans laquelle l'auteur des Confessions fait une relation détaillée de ce qui lui est arrivé en Angleterre. 'La relation de ces traitements abominables et des dangers qu'il a courus ayant été remise au ministère anglais, tout ce qui s'est passé à Wooton pendant les six derniers mois qui ont précédé son départ ou plutôt sa fuite,' est resté dans l'ombre, aussi Choumara somme-t-il le gouvernement britannique de la publier. La lumière sera faite alors: Rousseau et par contrecoup Choumara, seront lavés de l'accusation de folie.

Choumara reprend dès lors l'interprétation des faits qui le concernent. Lui aussi est une victime des Anglais qui ne lui pardonnent pas de les avoir attaqués dans ses Considérations militaires sur les mémoires $d u$ maréchal Suchet. C'est à ce livre, affirme-t-il, qu'est due la crise gouverne- 
mentale de juillet 1840 qui a vu la Russie, la Prusse, l'Autriche et l'Angleterre se coaliser et isoler la France dans la question d'Orient. Le signal de faire passer Choumara pour fou est venu de Londres, d'un journal Tory dévoué au duc de Wellington, et la police de Thiers lui a fait écho. Si on peut dans une certaine mesure apprécier certaines des intuitions de Choumara concernant Rousseau, on se rend compte de manière indéniable de sa folie lors de ces retours sur lui-même.

Pendant toute cette période qui va de 1840 à 1847, du Fou du roi à Théodore, Choumara continue de publier des traités sur les fortifications et des livres historiques. En 1847, il découvre un nouveau thème de recherche, puisqu'il édite cette année-là L'Astronomie simplifiée et perfectionnée par la correction d'une erreur capitale commise par les astronomes anciens et modernes, ou Examen critique raisonné de l'exposition du système du monde par Laplace. En 1855, c'est Véritable cause physique de la pesanteur des corps terrestres et de la gravitation universelle des corps célestes..., et, en 1864, Solution générale, simple, complète et pratique des magnifiques problèmes de la navigation aérienne.... Le dernier livre qui lui est attribué date d'un an avant sa mort: il s'agit de Coup d'oeil d'ingénieur militaire sur l'état actuel de l'Europe, ou introduction à la défense des Etats par la fortification, paru en 1869.Comme le note Queneau, 'Choumara, qui vécut dans les quatrevingt-quatre ans, paraît[...]sur ses vieux jours avoir délaissé ses persécuteurs pour s'occuper d'astronomie, de navigation aérienne et de politique extérieure. ${ }^{25}$

Vers 1840, c'est bien un fou littéraire qui lit Rousseau. Si l'on perçoit ici et là quelques éclairs qui offrent une interprétation originale, annonçant quelques-uns des commentaires modernes sur l'auteur des Confessions, on découvre à quel point ils sont perdus au sein d'un délire personnel qui leur ôte toute valeur. La lecture de Choumara est totalement influencée par son idée fixe de persécution, aussi se limite-t-elle essentiellement aux écrits autobiographiques, dans lesquels elle relève uniquement ce qui touche au complot. Elle est par ailleurs une lecture à usage strictement privé et ne peut, comme le disait Queneau, avoir aucun écho chez d'autres hommes. Rousseau manifestait dans les Dialogues son dessein de sortir du cercle où on l'enfermait et apportait maintes preuves de son équilibre et de sa vertu pour convaincre un lecteur de bonne foi. En Choumara, il trouve certes un lecteur convaincu de sa santé mentale, mais ce lecteur est fou. Si la preuve par Jean-Jacques a un sens pour Choumara, la preuve par Choumara n'a aucun sens pour Rousseau ni pour nous. 


\section{Notes}

1 J. Borel, Génie et folie de J.-J. Rousseau (Paris: Corti, 1966) 190.

2 Raymond Queneau, Les Enfants du limon (Paris: Gallimard, 1938) 121.

3 André Blavier, Les Fous littéraires (Paris: Veyrier, 1982) 12, n.3.

4 P.G. Brunet, Les Fous littéraires: essai bibliographique sur la littérature excentrique (Bruxelles: Gay et Doucé, 1880).

5 Queneau, ou plutôt Chambernac, le classe dans un chapitre intitulé 'Police secrète et mouchards' (251), Blavier dans celui intitulé 'Persécutés, persécuteurs et faiseurs d'histoire' (497-501).

6 Choumara, Un ingénieur militaire et la police parisienne, ou deux lettres à M.G. Delessert, préfet de police... (Paris: Imprimerie de Bourgogne et Martinet,1840) 28.

7 Citons quelques-uns de ces textes: Lettre à M. le baron Monnier, rapporteur de la Chambre des Pairs, chargé de l'examen du projet de loi sur les fortifications de Paris, 16 mars 1841 (Paris: 1841, 16pp.); Résumé historique des honteux échecs éprouvés par les armées britanniques depuis le commencement de la Révolution française jusqu'en 1814 (Paris: 1844, 30pp.); Leçon de fortification donnée à M. Arago, secrétaire perpétuel de l'Académie des Sciences, par le chef de bataillon de Génie Th. Choumara, pour servir de réponses aux lettres de M. Arago sur les fortifications de Paris (Paris: 1844, 48pp.).

8 Choumara, Le Fou du roi...(Paris: Imprimerie de Bourgogne et Martinet, 1841) 10.

9 En 1934 par exemple, après les événements du 6 février et l'affaire Stavisky, Léon Daudet dénonce dans $L^{\prime}$ Action française les rapports que la Sûreté générale entretient avec la pègre, notamment avec la célèbre maison close Le Sphinx et le cabaret Le Frolic's: voir L'Action française des 27, 31 mars et 10 avril 1934.

10 Choumara n'est pas le seul à établir alors un rapprochement entre les jésuites, les Anglais et la police secrète. On le trouve également chez un autre fou littéraire, Jean-Baptiste Gautrin, qui édite en 1846, une feuille autographiée, La Police secrète, études machiavéliques, dans laquelle il développe ce thème. Sur cette époque particulièrement hostile aux jésuites, on pourra lire l'ouvrage de Jean Lacouture, Jésuites. Une multibiographie. 2: Les revenants (Paris: Seuil, 1992) 75-113.

11 Claude Wacjman, 'Sur la maladie de Rousseau: témoins et interprètes,' Etudes J.-J. Rousseau 6 (1992): 109-110.

12 Le Fou du roi, 57, n.1. Les italiques sont dans le texte.

13 Wacjman 101.

14 Le Fou du roi 5-6. Les italiques sont dans le texte.

15 Choumara, Théodore, ou cinquante-neuf ans de la vie d'un homme de tête et de coeur... (Paris: Chez les marchands de nouveautés, 1847) CI.

16 Le Fou du roi 6.

17 On trouve ce tract à la Bibliothèque nationale de Paris sous la cote $8^{\circ} \operatorname{Ln} 27.28705$. Signalons en passant qu'une littérature existe sur ce thème du sosie de Rousseau: on connaît l'anecdote du tailleur de Sophie Arnoud rapportée par Musset-Pathay dans Histoire de la vie et des ouvrages de J.-J. Rousseau (Paris, 1821) t.1:181-182. Voir aussi Ed. Gachot, 'Un sosie de Jean-Jacques,' Le Magasin pittoresque, 1910, t.XI, série 3. Les médecins des hopitaux rapportent aussi parfois des exemples de fous 
ayant lu Rousseau: P. Garnier, La Folie à Paris (Paris, 1890) 221; Génil-Perrin, Les Paranoïaques (Paris, 1926) 317.

18 Jean Roussel, 'Le Phénomène de l'identification dans la lecture de Rousseau,' Annales de la Société J.-J. Rousseau 39 (1980): 65-77.

19 Roussel 72.

20 J.-J. Rousseau, Confessions, Oeuvres complètes, Bibliothèque de la Pléiade, t.1:279. Choumara cite ce texte dans Théodore, XCIX-C, et le place en épigraphe au chapitre sur Rousseau dans Le Fou du roi 53.

21 Rousseau, Dialogues 712; Choumara, Théodore, C.

22 Le Fou du roi 13-14. Les italiques sont dans le texte.

23 Fou 82. Les italiques sont dans le texte.

24 Fou 93.

25 Queneau 253. 